\title{
THz Spectroscopy with a Holographic Fourier Transform Spectrometer Plus Array Detector Using Coherent Synchrotron Radiation
}

\author{
N. I. Agladze, ${ }^{1}$ J. M. Klopf, ${ }^{2}$ G. P. Williams, ${ }^{2}$ and A. J. Sievers ${ }^{1, *}$ \\ ${ }^{1}$ Laboratory of Atomic and Solid State Physics, Cornell University \\ Ithaca, NY 14853-2501, USA \\ ${ }^{2}$ Free Electron Laser Facility, Jefferson Lab, 12000 Jefferson Avenue, \\ Newport News, VA 23606, USA \\ *Corresponding author: sievers@ccmr.cornell.edu
}

\begin{abstract}
Using coherent $\mathrm{THz}$ synchrotron radiation a holographic Fourier transform spectrometer coupled to an array detector was tested experimentally to determine its viability as a spectral device. Somewhat surprisingly the overall performance strongly depends on the absorptivity of the birefringent lithium tantalate pixels in the array detector.
\end{abstract}

OCIS codes: 120.6200, 300.6340, 260.3160, 300.6300. 
With the development of coherent broadband THz synchrotron sources based on the radiation produced by short bunches of electrons in synchrotron orbits[1] interest has developed in the appropriate spectroscopic instrumentation for short time measurements[2, 3]. In addition to time dependent spectroscopy there is also interest in using this $\mathrm{mm}$ and submillimeter wave radiation signature for dynamical analysis of a short electron bunch. Spectroscopic measurement of the bunch length by means of its coherent radiation signature becomes technically easier as the bunch gets shorter since the resulting radiation extends over a larger frequency interval. The determination of the bunch form factor using this technique has been described in some detail[48].

A holographic Fourier transform spectrometer (HFTS) has no moving parts and produces an interferogram in the spatial, not in the time domain. Thanks to its high throughput, HFTS have found use in the remote and time dependent sensing where they outperform even multichannel spectrographs[9]. The extremely broad spectral coverage of an HFTS would be particularly beneficial in the $\mathrm{THz}$ region where a diffraction grating cannot be used without elaborate optics for filtering out higher diffraction orders. But the large interference angle required for a THz HFTS makes associated field aberrations too large for a realistic size of the array detector. This difficulty has been resolved by dividing the Fourier optics into two halves with a tilt interferometer design[10]. Here we present experimental tests of this new THz HFTS instrument using the coherent synchrotron radiation produced in the energy recovery linac of the free electron laser facility at Jefferson Laboratory.

A three dimensional schematic of the THz HFTS optical design is shown in Fig. 1. The fore optics consisting of a $45^{\circ}$ off-axis parabolic mirror accepts the collimated THz radiation. The intermediate focus is formed slightly behind a wire grid beamsplitter, which also serves as 
the field angle-limiting aperture. The motor driven shutter in each arm provides the means to measure the intensity in each of the interferometer arms. The two tilted mirrors direct the beams towards the $30^{\circ}$ off-axis parabolic mirrors, which serve as the mirror analog of the Fourier transform lens. Finally the collimated beams converge on the array detector located with its plane perpendicular to the axial beams bisector. An additional wire grid polarizer is located next to the detector (not shown) in order to mix the orthogonal polarizations propagating along the interferometer arms. Assuming the amplitude transmission and reflection coefficients are $\tau$ and $1-\tau$ at the beamsplitter for a specific polarization analyzed by the detector, then the intensity produced in the detector plane $(x y)$ by beams along the axial directions is given by the following relation [10]

$$
I(x, y)=\int_{0}^{\infty} I^{0}(\sigma, x, y)\left\{\tau^{2}+(1-\tau)^{2}+2 \tau(1-\tau) \cos [(2 \pi \sigma y) 2 \sin (\alpha / 2)]\right\} B(\sigma) d \sigma,
$$

where $I^{0}(\sigma, x, y)$ is the intensity at frequency $\sigma \equiv 1 / \lambda$ ( $\lambda$ is the wavelength) in the absence of the beamsplitter. The factor $2 \sin (a / 2)$ in the cosine argument determines the scale of the interference pattern. This scaling factor determines the spectral resolution and the high frequency cutoff of the instrument for a given size and pixel dimension of the array detector. The only commercial array detector available today for the THz region is the Pyrocam III and it was used in these experiments. Its sensitivity is sufficiently low that this instrument could not be tested with incoherent sources of THz radiation such as a globar or mercury arc. All experimental tests of the new spectrometer were performed using coherent $\mathrm{THz}$ synchrotron radiation.

Synchrotron radiation produced by short electron bunches in one of the bending magnets was delivered through the vacuum optical transmission line into the radiation shielded laboratory. Figure 2 shows the actual arrangement. After the diamond exit window (1) the THz 
radiation propagated in air and it is collimated with a $90^{\circ}$ parabolic reflector ( 2 ). The original horizontal polarization of the synchrotron radiation after reflections in the transfer line appeared at the optical table at a small angle from the vertical direction. (It is referred to as horizontal in the following.) For measurements of transmission spectra an intermediate focus was created between a pair of additional $90^{\circ}$ parabolic mirrors (3) and (4). The tilt interferometer (7) is located in the collimated beam and the array detector (8) is installed on a translation stage so that the center burst of the static interference pattern produced by the interferometer can be positioned at any pixel of the array.

In order to compensate for the non-uniform illumination of the detector area the following calibration procedure was used. Three separate measurements were performed for various positions of the shutters blocking the beam in each arm of the interferometer (see Fig. 1). First, background intensity distribution $I_{0}(x, y)$ in the array was measured with both shutters closed. Then two intensities $I_{1}(x, y)$ and $I_{2}(x, y)$ were measured for shutters blocking only one of the interferometer arms. Distributions $I_{1}(x, y)-I_{0}(x, y)$ and $I_{2}(x, y)-I_{0}(x, y)$ with the scattered parasitic light subtracted for both arms of the interferometer were added and normalized to give the correction factor $k(x, y)$ between 0 and 1 . The corrected intensity distribution $I_{c}(x, y)$ was calculated from the signal $I(x, y)$ with both shutters opened according to the following relation

$$
I_{c}(x, y)=\left[I(x, y)-I_{0}(x, y)\right] / k(x, y)
$$

The same correction factors were used for the reference and for the sample spectra during subsequent transmission measurements. This procedure ignores any frequency dependence of the 
beamsplitter efficiency and assumes that the intensity distributions produced by the two interferometer arms differ only by a certain factor. Generally the latter point is not true for a polarizing beamsplitter with different intensity distributions for horizontal and vertical polarizations of the synchrotron radiation. In order to avoid this problem an additional polarizer transmitting only horizontal polarization was installed before the interferometer.

Measurements were performed in the quasi-continuous mode using the internal chopper of the Pyrocam detector interrupting the incoming THz beams at $48 \mathrm{~Hz}$. A laptop computer running LabVIEW software controlled operation of the shutters and of the array detector. Spectra were calculated and displayed in real time. Two positions were used for the center burst of the spatial interferograms. When the maximum possible resolution was desired it was located at the 10th pixel, and when a better phase correction was required it was located at the 20th pixel from the side. The Pyrocam III detector has 124 X 124 square pixels with the pitch $100 \mu \mathrm{m}$ and the resulting spectral resolution is $1.2-1.3 \mathrm{~cm}^{-1}$ and the maximum non-aliased frequency is 114 $\mathrm{cm}^{-1}$.

Examples of corrected static interference patterns and corresponding calculated spectra are shown in Fig. 3. Notable are the periodic oscillations in the spectra. These measurements were performed with following parameters of the energy recovery linac: current $1 \mathrm{~mA}$, bunch charge 100 pC, repetition rate 9.36 MHz, beam energy 114.65 MeV. The Pyrocam III accumulation time was 1 second. Detuning of the bunch energy by changing the gradient field in the RF cavities varied the electron bunch length. The sharpest center burst is observed for the -70 $\mathrm{keV}$ detuning, hence the narrowest electron bunch is observed at this beam energy.

In order to determine the spectral response of the array detector it was used together with a scanning Michelson interferometer to analyze the same synchrotron radiation. Both the 
scanning Michelson and the HFTS spectral results are shown in Fig. 4 with dot-dash and solid lines, respectively. Apart from a difference in resolution the two spectra are quite similar confirming that the non-uniform spectral response of the THz HFTS measurement is due to the detector array. For an additional comparison the same spectral region was measured with the Golay cell in the scanning Michelson interferometer (dashed line in Fig. 4). Crossed wire grid polarizers were used to attenuate the radiation to the level acceptable for the Golay cell. The most probable explanation of the spectral non-uniform characteristics of the array detector is the production of a channel spectrum in its pixels. The measured period $15 \mathrm{~cm}^{-1}$ corresponds to a 50 $\mu \mathrm{m}$ thickness for the lithium tantalite used in the detector elements. Note that similar nonuniform spectral response due to a channel spectrum has also been reported for in some single element pyroelectric detectors [8].

To examine the high frequency characteristics of the THz HFTS a transmission spectrum of a band pass THz filter (QMC Instruments) was measured as well. The results for the THz HFTS are compared with those from a lamellar interferometer with a Golay detector in Fig. 5. The angular distribution of the coherent synchrotron radiation is wavelength dependent due to the electron beam vacuum chamber screening effects[11]. For the effective focal length $10 \mathrm{~cm}$ used here the angular distribution was sufficiently narrow at the filter transmission maximum (28 $\mathrm{cm}^{-1}$ ) so that the collimated beam did not cover the whole area of the array detector at this frequency. This resulted in the effective degradation of the spectral resolution in the THz HFTS but the general shape of the band pass filter spectrum is similar for both kinds of spectrometers. For a more detailed spectral test of the HFTS plus array detector the channel spectrum of a silicon slab was also measured and compared with that determined from a lamellar interferometer plus Golay measurement. The results are shown in Fig. 6. The silicon wafer had 
only one side polished; the other surface was rough with a characteristic grain size $\sim 50 \mu \mathrm{m}$, determined with the aid of an optical microscope. Only a two pass interference spectrum results. The equation that describes the silicon wafer spectrum fit with one two-pass interference beam is

$$
T(\sigma)=A+C \cos (4 \pi \sigma n d)
$$

Here $d=401 \mu \mathrm{m}$ is the wafer thickness, $n=3.42$ is the silicon refractive index at $\mathrm{THz}$ frequencies determined from the fit of the lamellar spectrum, $A$ sets the average transmission value, $C$ models the strength of the channel spectrum. Fitting parameters are shown in Table 1. Value of $A$ matches the transmission of the silicon slab with only two reflections taken into account. This spectrum is represented by the dotted trace in Fig. (7). Comparison with the lamellar data in the figure show that irregular frequency shifts appear in the THz HFTS spectrum. These frequency shifts are consistent with a beating between two different silicon channel spectra. One spectrum is described by Eq, (3) and the second one has a different scale resulting from the beams incident on the array at a slightly different angle. The result is Eq. (4).

$$
T(\sigma)=A+C\{B \cos (4 \pi \sigma n d)+(1-B) \cos [4 \pi \sigma n n \sin (\alpha / 2) / \sin (\alpha / 2+\delta)]\}
$$

where $\delta$ is the change of the angle transmitted through the pixels, B describes the relative strength of the beating spectra, $\alpha=24.4^{\circ}$ is the tilt interferometer designed angle. Fitting parameters are listed in Table 1 . The poor fit near $15 \mathrm{~cm}^{-1}$ is due to the large error in the transmission spectrum as a result of the weak sensitivity of the detector at this frequency. Neither angular distribution of the radiation at the silicon sample nor angular dependence of the scaling factor of the THz HFTS can account for the second scaling factor. 
How can one account for an extra angle? It is clear from Fig. 4 that multiple reflections occur within the pixel array demonstrating that the $\mathrm{THz}$ radiation passes through the lithium tantalate pixels. Due to the birefringence of the pixel material and polarization mixing inside the detector structure a beam coming from an interferometer arm can split into two beams with a few degrees difference. After reflection from the back metal plate these beams will fall on the pixels from the backside again and will produce a rescaled interferogram component. This change in angle will result in scaling factors $2 \sin (\alpha / 2)$ and $2 \sin (\alpha / 2+\delta)$ for the incident and reflected beam paths, respectively. The fitted angle $\delta=2.3^{\circ}$ is compatible with the expected lithium tantalate birefringence effects. This simple model is not a substitute for the rigorous treatment taking into account the near field effects in the real 3D array detector structure. Such a treatment is not possible because of proprietary restrictions for the array details; however, the model illustrates what happens when the array is not properly designed for spectral measurements at $\mathrm{THz}$ frequencies.

To optimize this spectroscopic system at least two changes in the array detector fabrication are recommended. The pixels should be vacuum impedance matched in the $\mathrm{THz}$ region to increase the sensitivity and an efficient absorber should be placed behind the pixels to avoid complications due to back reflections of the beams. Since the pixel spacing in the array sets the shortest wavelength limit of the HFTS the pixel size should be reduced so that a broader spectral range can be covered.

Summarizing, the experimental test of the holographic FTS for the THz region demonstrate the following.

(1) A new spectrometer design for the THz, the HFTS built around the tilt interferometer, using an array detector and coherent synchrotron radiation has been demonstrated. 
(2) A qualitative characterization of the electron bunch length with the new instrument is shown.

(3) These experimental tests of the new spectrometer demonstrate its viability as a spectral device and reveal that its performance is strongly dependent on the details of the array detector construction.

Acknowledgments

Financial support from the Department of Energy (grant DOE-DE-FG02-

04ER46154). Use of facilities of the Cornell Center for Material Research (supported by NSF grant DMR 0520404) is also acknowledged. 


\section{References}

1. T. Nakazato, M. Oyamada, N. Niimura, S. Urasawa, O. Konno, A. Kagaya, R. Kato, T. Kamiyama, Y. Torizuka, T. Nanba, Y. Kondo, Y. Shibata, K. Ishi, T. Oshak, and M. Ikezawa, "Observation of Coherent Synchrotron Radiation," Phys. Rev. Lett. 63, 12451248 (1989).

2. G. L. Carr, M. C. Martin, W. R. McKinney, J. Jordan, G. R. Neil, and G. P. Williams, "High-power terahertz radiation from relativistic electrons," Nature 420, 153-156 (2002).

3. G. P. Williams, "Filling the THz gap - high power sources and applications," Rep. Prog. Phys. 69, 301-326 (2006).

4. Y. Shibata, T. Takahashi, T. Kanai, K. Ishi, M. Ikezawa, J. Ohkuma, S. Okuda, and T. Okada, "Diagnostics of an electron beam of a linear accelerator using coherent transition radiation," Phys. Rev. E 50, 1479-1484 (1994).

5. R. Lai and A. J. Sievers, "On using the coherent far IR radiation produced by a chargedparticle bunch to determine its shape .1. Analysis," Nucl. Instr. Meth. Phys. Res. A 397, 221-231 (1997).

6. G. Schneider, M. G. Billing, R. Lai, and A. J. Sievers, "Comparison of electron bunch asymmetry as measured by energy analysis and coherent transition radiation," Phys. Rev. E 56, R3780-R3783 (1997).

7. T. Watanabe, J. Sugahara, T. Yoshimatsu, S. Sasaki, Y. Sugiyama, K. Ishi, Y. Shibata, Y. Kondo, K. Yoshii, T. Ueda, and M. Uesaka, "Overall comparison of subpicosecond electron beam diagnostics by the polychromator, the interferometer and the femtosecond streak camera," Nucl. Instr. Meth. Phys. Res. A 480, 315-327 (2002). 
8. D. Mihalcea, C. L. Bohn, U. Happek, and P. Piot, "Longitudinal electron bunch diagnostics using coherent transition radiation," Phys. Rev. ST Accel. Beams 9, 08280110828017 (2006).

9. N. I. Agladze and A. J. Sievers, "Miniaturization of holographic Fourier-transform spectrometers," Appl. Opt. 43, 6568-6579 (2004).

10. N. I. Agladze and A. J. Sievers, "Holographic Fourier transform spectrometer for THz region," Opt. Lett. (March 1, 2010).

11. S. Kheifets and B. Zotter, "Shielding Effects on Coherent Synchrotron Radiation," in Micro Bunches Workshop, E. B. Blum, M. Dienes, and J. B. Murphy, eds. (AIP, Woodbury, 1996), p. 424. 
Table 1. HFTS channel spectrum fitting parameters.

\begin{tabular}{|c|c|c|c|c|}
\hline Fit function & $A$ & $B$ & $C$ & $\delta$ \\
\hline Eq. (3) & $0.469 \pm 0.007$ & - & $0.07 \pm 0.01$ & - \\
\hline Eq (4) & $0.466 \pm 0.007$ & $0.43 \pm 0.08$ & $0.10 \pm 0.01$ & $2.3^{\circ} \pm 0.1^{\circ}$ \\
\hline
\end{tabular}




\section{Figure Captions}

Figure 1. Optical setup of the tilt interferometer based holographic FTS. 1 - fore optics ( $45^{\circ}$ offaxis parabolic mirror); 2 - beamsplitter; 3,4 - shutters; 5,6 - out-of-plane tilted mirrors; 7,8 two halves of the split Fourier transform system ( $30^{\circ}$ off-axis parabolic mirrors); 9 -the array detector.

Figure 2. Experimental setup at JLab for testing THz HFTS. Foreground: The THz radiation is delivered from the accelerator through the vacuum transfer line and is focused on the output diamond window (1). The white line identifies the THz radiation optical path. It is collimated by the parabolic reflector (2). An intermediate focus for sample transmission measurements is formed between the two parabolic reflectors (3) and (4). Mirror (5) sends the collimated beam to the interferometer (7). The interference pattern is analyzed with the pyroelectric array detector (8). Background: Removable mirror (6) is used to send the THz beam to a separate experimental setup (9).

Figure 3. Coherent synchrotron radiation interference patterns for different electron bunch lengths and corresponding coherent synchrotron spectra. The larger the frequency interval of the spectrum the shorter is the bunch. The Pyrocam array has a highly non-uniform spectral response due to the channel spectrum produced in the elements.

Figure 4. Comparison of coherent synchrotron spectra measured with a traditional scanning Michelson interferometer and for the THz HFTS. Broken line: THz HFTS; dotted line: 
Michelson interferometer with the same detector; solid line: Michelson interferometer with a Golay cell.

Figure 5. Transmission spectrum of a THz band pass filter (QMC Instruments). Solid line: spectrum measured with THz HFTS; dashed line: spectrum measured with a lamellar scanning FTS.

Figure 6. Transmission spectrum of a silicon slab. Solid line: spectrum measured by THz HFTS; dashed line: spectrum measured with a lamellar scanning FTS.

Figure 7. THz HFTS transmission spectrum of the silicon wafer compared with two models. HFTS transmission spectrum (solid line); fits to single scale model channel spectrum (dotted line); fit to the superposition of two channel spectra with the different scales (broken line). 


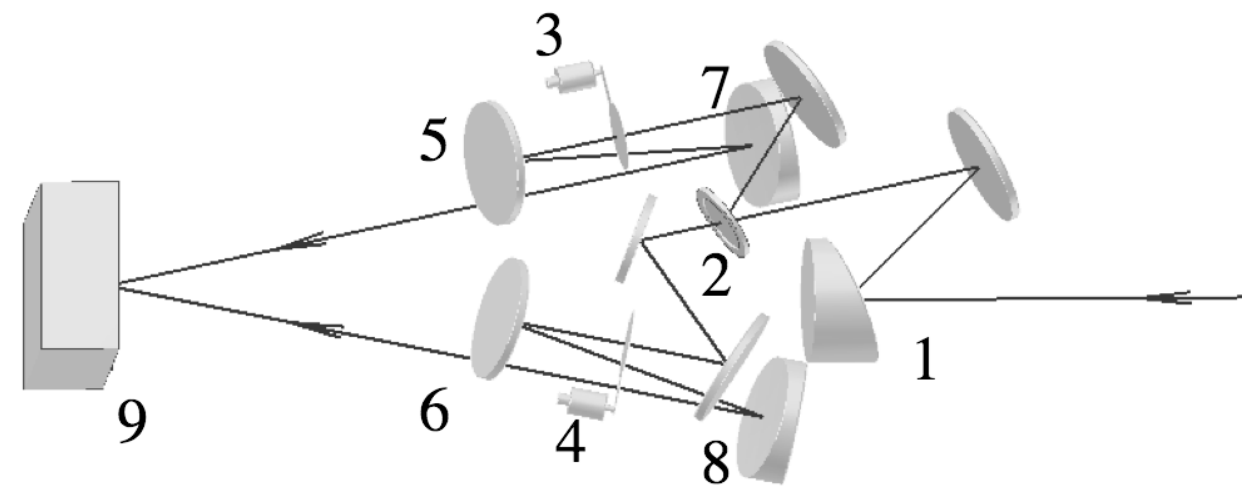

Figure 1. 


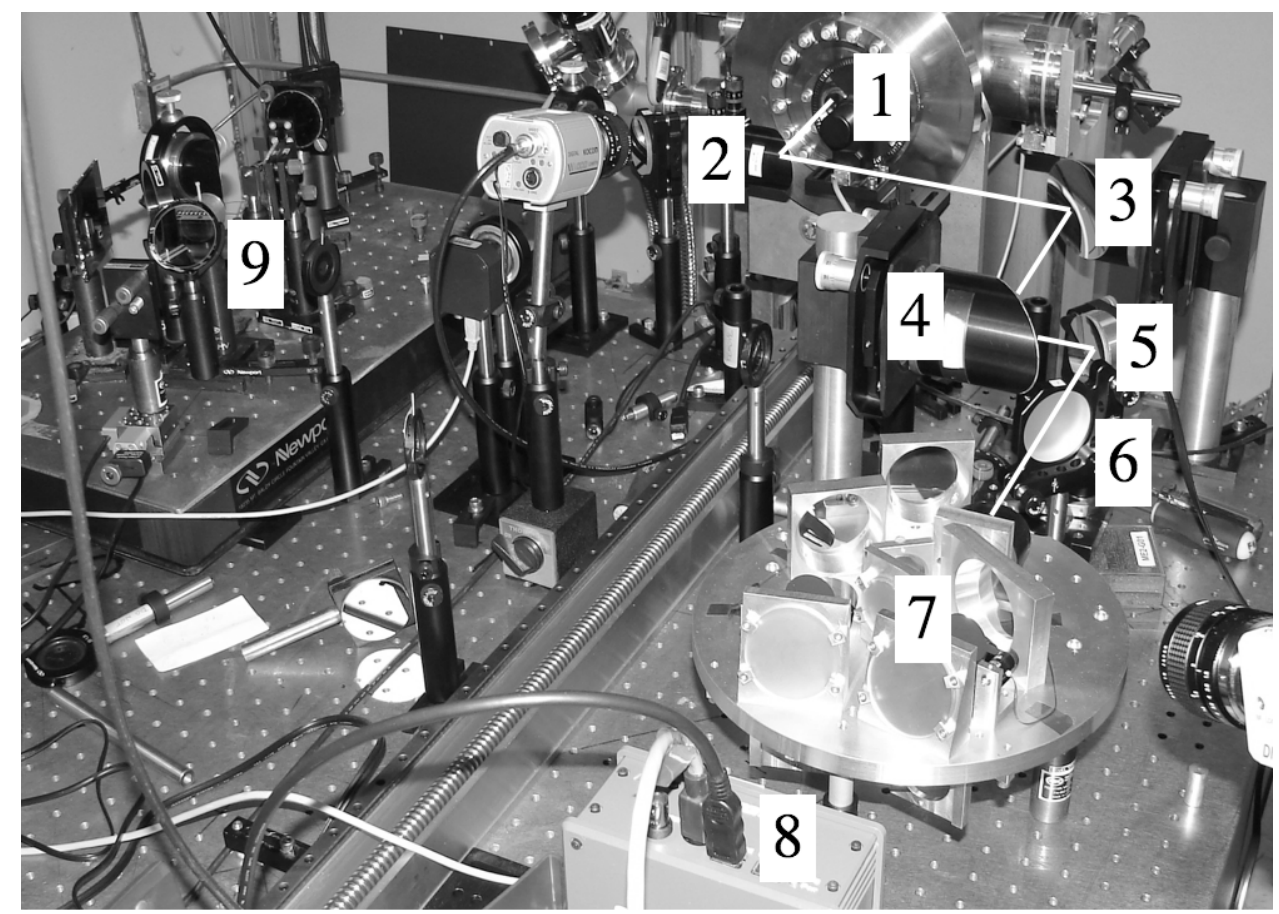

Figure 2. 

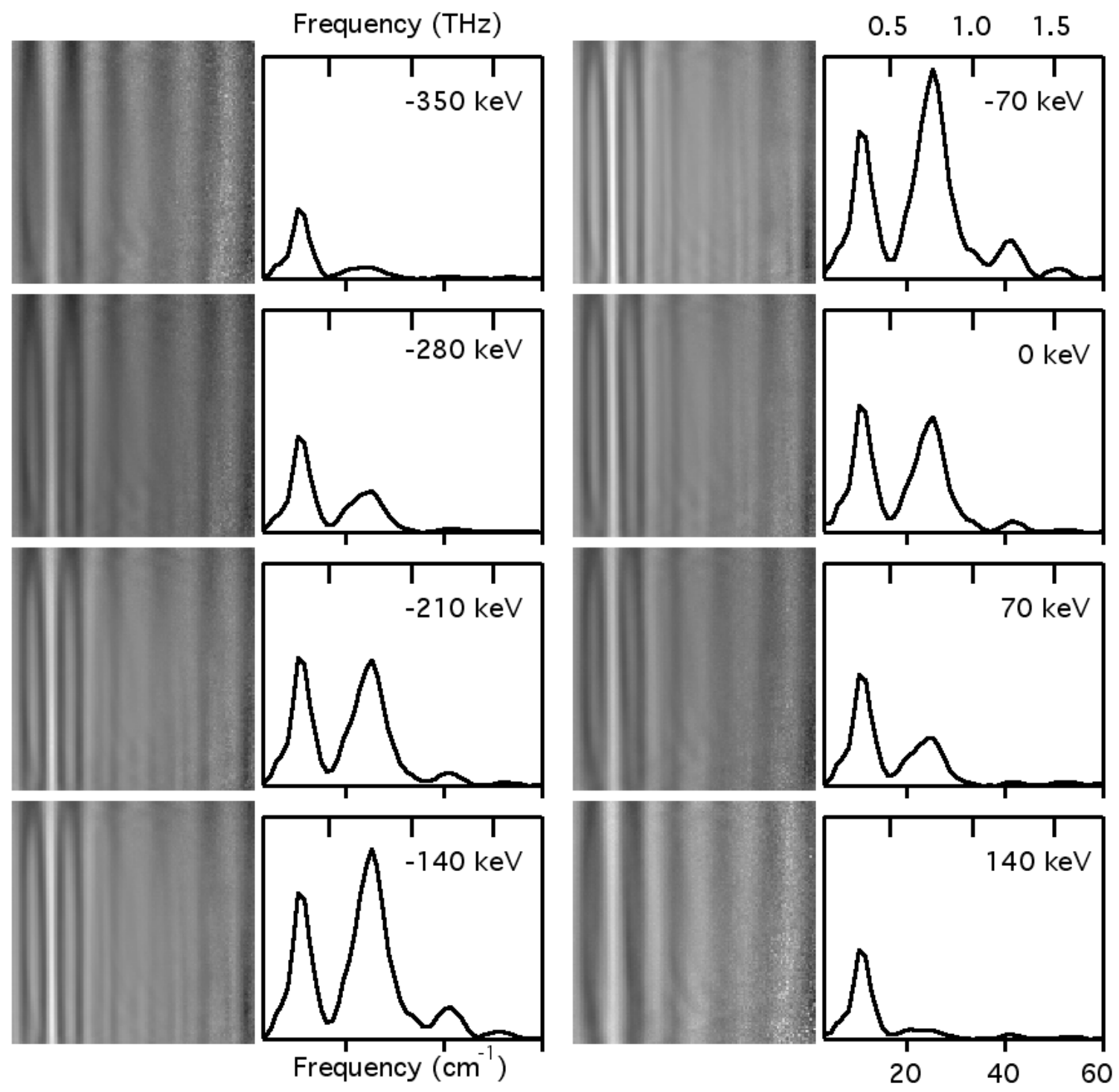

Figure 3. 


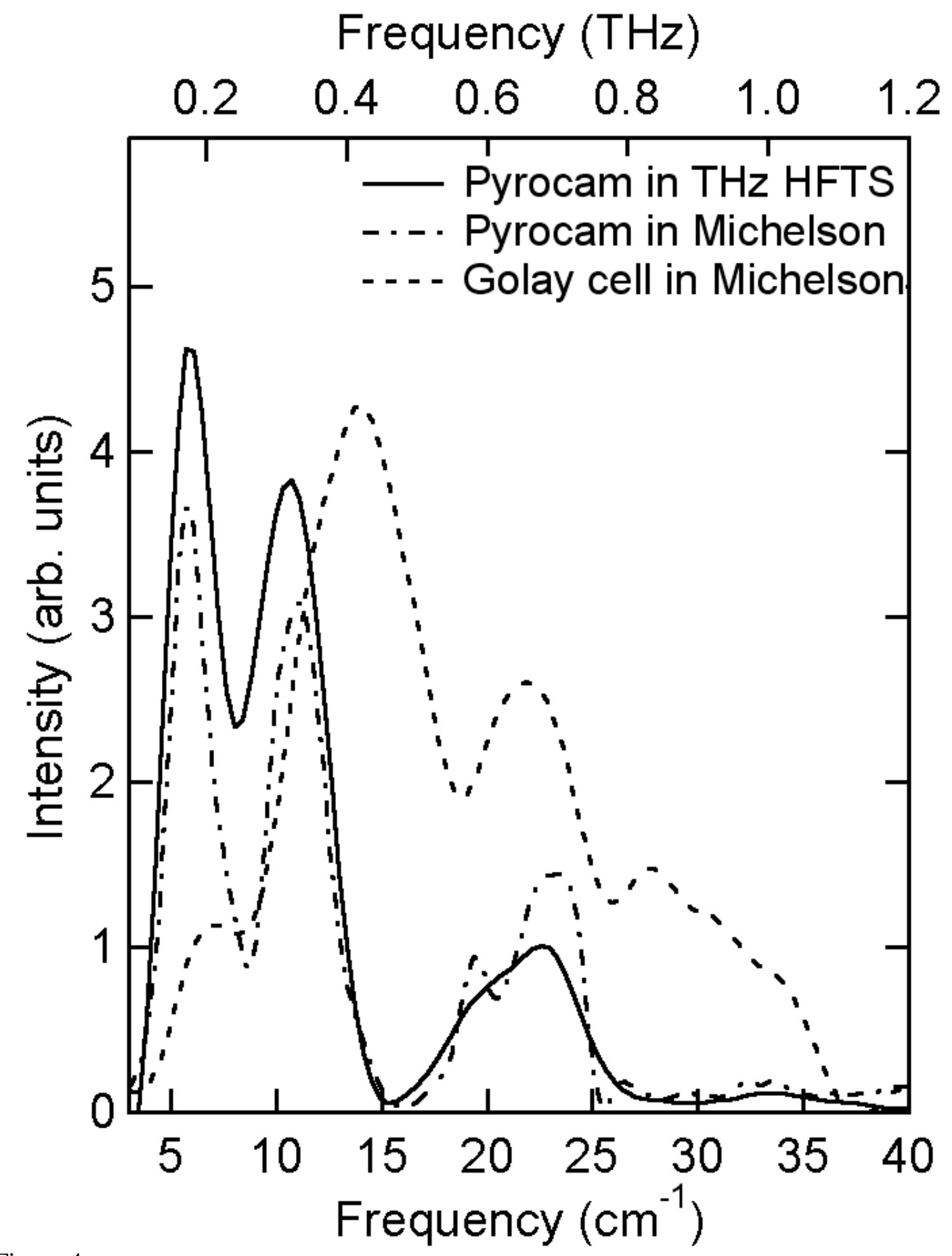

Figure 4. 


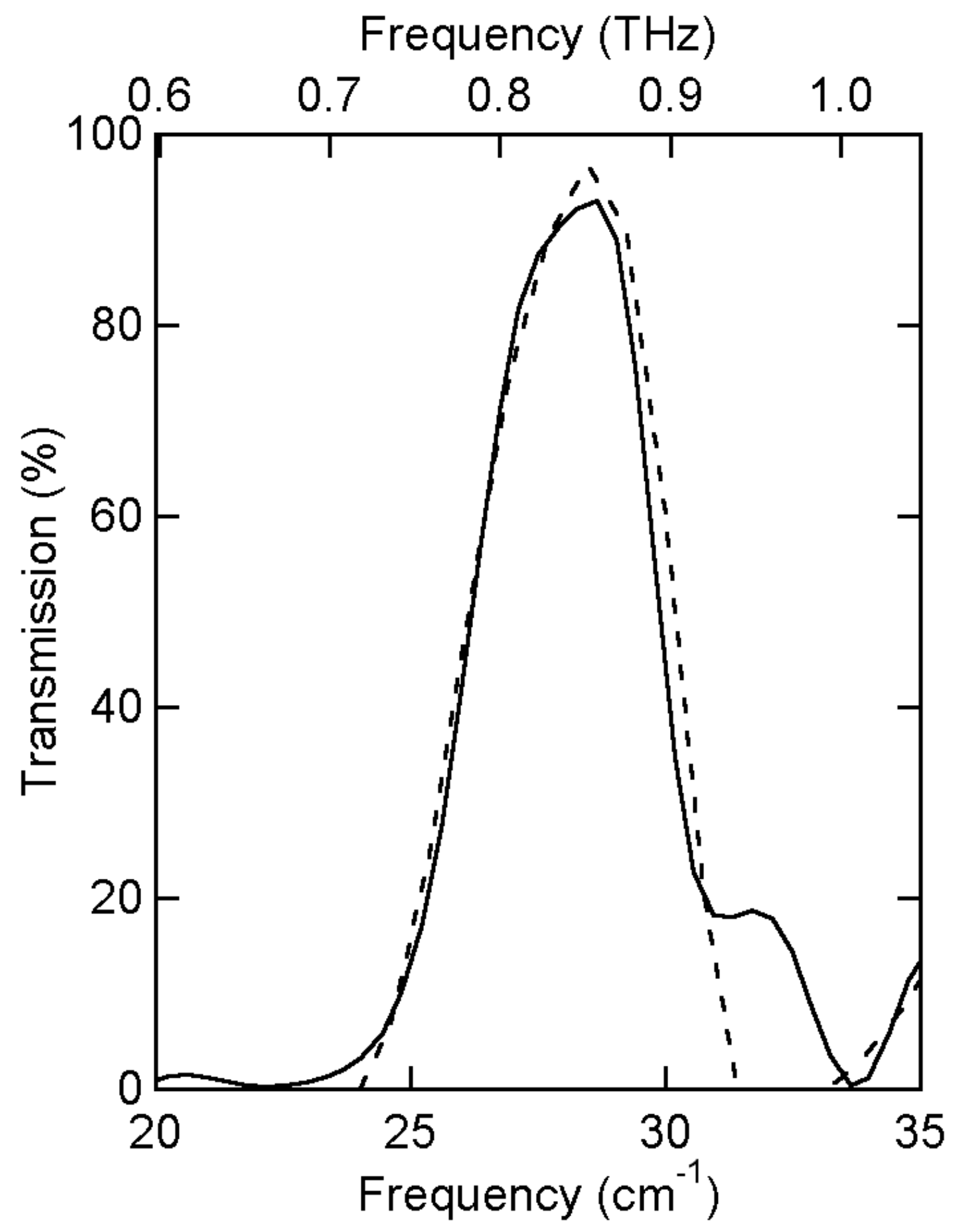

Figure 5. 


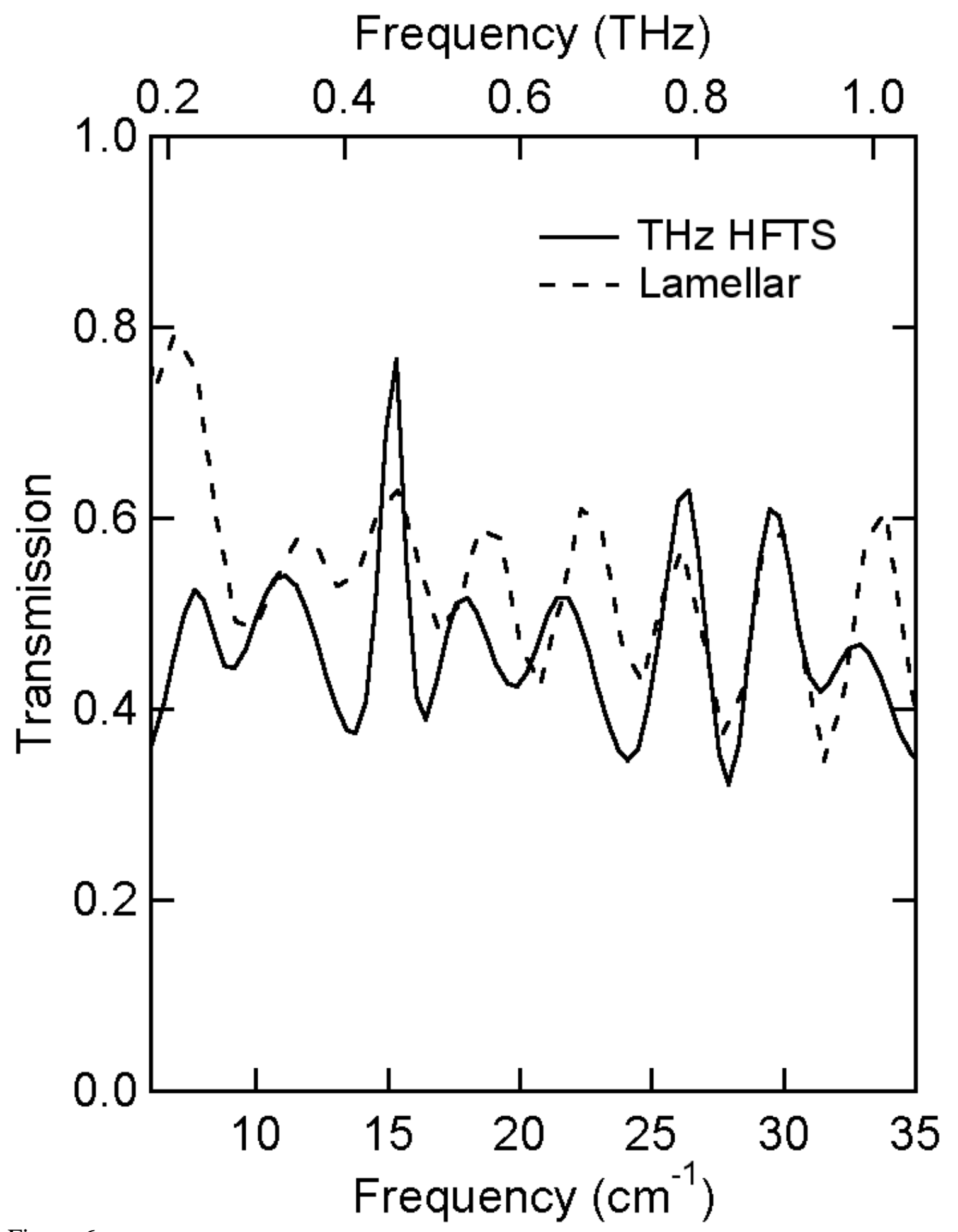

Figure 6. 


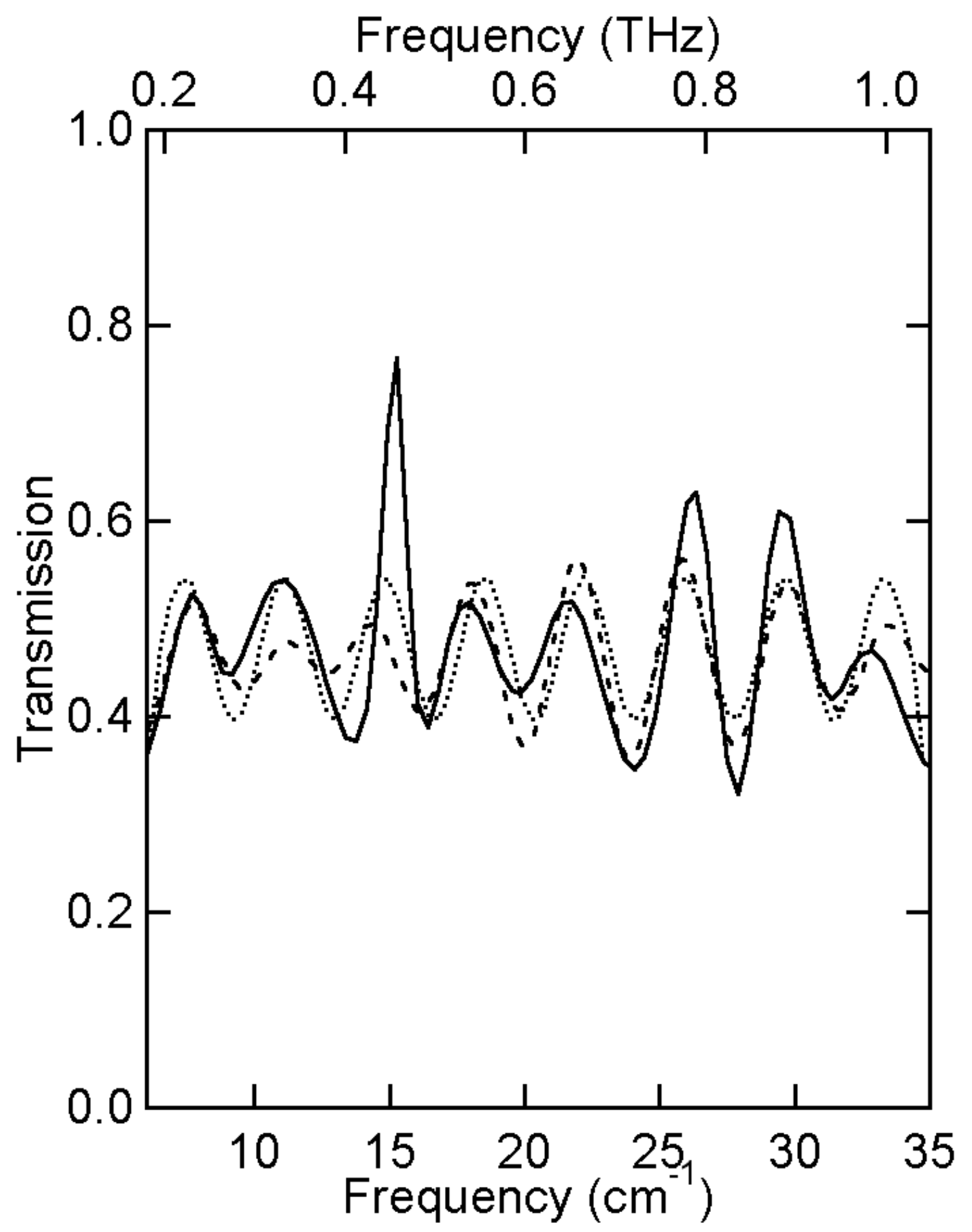

Figure 7. 